\title{
(1-naftilmetiliden)izonikotinohidrazid molekülünün yapısal ve elektronik özelliklerinin teorik olarak incelenmesi
}

\author{
Güventürk UĞURLU1 ${ }^{1}$, Hacali NECEFOĞLU²
}

ÖZET: Bu çalışmada, (1-naftilmetiliden)izonikotinohidrazid molekülünün (I) kararlı konformerlerini belirlemek için, çalışılan molekülün konformasyon analizi Yoğunluk Fonksiyonel Teorisi (DFT/B3LYP) metodu ile $6-311++G(d, p)$ temel seti kullanılarak incelendi. Bu molekülün iki kararlı durum konformerleri (C-I ve C-II) bulundu ve bu konformerler Hartree Fock HF/6-311++G(d,p) ve B3LYP/6-311++G(d,p) teori düzeyinde ve temel seti ile optimize edildi. Hesaplama sonuçları, I molekülünün en kararlı konformerinin C-I olduğunu gösterdi. Taban durumundaki C-I ve C-II konformerler için, dipol moment $(\mu)$, polarizebilite $(\alpha)$, birinci-derece hiperpolarizebilite $(\beta)$, en yüksek dolu molekül orbital enerjileri $\left(\mathrm{E}_{\mathrm{Hомо}}\right)$, en düşük boş molekül orbital enerjileri ( $\left.\mathrm{E}_{\mathrm{LUMO}}\right)$ ve enerji fark1 $\left(\Delta \mathrm{Eg}=\mathrm{E}_{\mathrm{LUMO}}-\mathrm{E}_{\mathrm{HOMO}}\right)$ her iki modelde $6-311++\mathrm{G}(\mathrm{d}, \mathrm{p})$ taban seti kullanılarak hesaplanmıştır. Bu enerjiler dikkate alınarak sertlik $(\eta)$ ve elektronegatiflik $(\chi)$ parametreleri hesaplandı. Ayrıca, elde edilen optimize yapı ile GIAO yöntemine göre ${ }^{1} \mathrm{H}-\mathrm{NMR}$ ve ${ }^{13} \mathrm{C}$-NMR kimyasal kayma değerleri B3LYP / $311++\mathrm{G}(2 \mathrm{~d}$, p) ve Hartree Fock HF / 6-31G teori düzeyinde hesaplandı. C-I ve C-II konformerleri için dipol moment değerleri, sırasıyla B3LYP / 6-311 ++ G (d, p) ile 2.05 ve 2.32 ve HF / 6-311 ++ G (d, p) 1.94 ve 2.32 Debye bulundu. I molekülünün yapisal parametreleri, literatürdeki verilerle karşılaştırıldı.

Anahtar Kelimeler: DFT/B3LYP, HF, hiperpolarizebilite, (1-naftilmetiliden)izonikotinohidrazid, polarizebilite.

\section{Theoretical investigation on the structural and electronic properties of (1-naphthylmethylidene)isonicotinohydrazide molecule}

\begin{abstract}
In this paper, in order to determine the stable conformers of (1-naphthylmethylidene) isonicotinohydrazide molecule (I), the conformational analysis of the molecule studied was investigated by Density Functional Theory (DFT/B3LYP) levels of theory using 6-311++G $(\mathrm{d}, \mathrm{p})$ basis set. The two stable states conformers (C-I and C-II) of the this molecule have been found and these conformers were optimized HF/ 6-311++G(d,p) and B3LYP/ 6-311++G(d,p) levels of theory and basis set. The computational results have shown that the most stable conformer of molecule I was as the C-I form. The dipole moment $(\mu)$, polarizability $(\alpha)$ and first order hyperpolarizability $(\beta)$, the highest occupied molecular orbital energies $\left(\mathrm{E}_{\text {номо }}\right)$, the lowest unoccupied molecular orbital energies $\left(\mathrm{E}_{\mathrm{LUMO}}\right)$ and energy gap $\left(\Delta \mathrm{Eg}=\mathrm{E}_{\text {LUмо }}-\mathrm{E}_{\text {номо }}\right)$ of C-I and C-II conformers have been calculated by using $6-311++\mathrm{G}(\mathrm{d}, \mathrm{p})$ basis set for both models at the ground state. The hardness $(\eta)$ and electronegativity $(\mathrm{x})$ parameters were determined taking into account these energy values. Besides, ${ }^{1} \mathrm{H}-\mathrm{NMR}$ and ${ }^{13} \mathrm{C}-\mathrm{NMR}$ chemical shift values according to the method GIAO by obtained the optimized structure were calculated using B3LYP $/ 311++\mathrm{G}$ (2d,p) and HF/6-31G levels of theory. The dipole moment values for C-I and C-II conformers are calculated at 2.05 and 2.32 with DFT/B3LYP level of the theory $6-311++\mathrm{G}(\mathrm{d}, \mathrm{p})$ basis set and at the HF/6-311++G(d,p) 1.94, and 2.32 Debye, respectively. The structural parameters of molecule I compared with data in the literature.
\end{abstract}

Keywords: DFT/B3LYP, HF, hyperpolarizability, (1-naphthylmethylidene)isonicotinohydrazide, polarizability

Güventürk UĞURLU (0000-0003-4171-7879), Kafkas Üniversitesi, Fen Edebiyat Fakültesi, Fizik Bölümü, Kars, Türkiye

Hacali NECEFOĞLU (0000-0003-2901-3748), Kafkas Üniversitesi, Fen Edebiyat Fakültesi, Kimya Bölümü, Kars, Türkiye Sorumlu yazar/Corresponding Author: Güventürk UĞURLU, gugurlu@kafkas.edu.tr 


\section{GİRIŞ}

Tüberküloz olarak adlandırılan bakterilerden kaynaklanan verem hastalığından yılda milyonlarca insan ölmektedir (Bloom et al., 1992; Sriram et al., 2009). XX. yüzyılın sonlarından itibaren mikrobiyal enfeksiyonların görülme sıklığının antimikrobiyal direnç sonucu arttı̆̆ müşahede edilmektedir (Judge et al., 2012; Sbarbaro, 1997). Bunedenle verem tedavisinde kullanılan ilaçların yetersiz olması, bu ilaçlara direnç geliştirmiş patojenik mikroorganizmalara karşı etkin yeni sınıf bileşikler arayışı ve sentezi önem kazanıyor. B3 vitamini gibi bilinen nikotinik asidin isomeri olan izonikotinik asidin hidrazidi-izoniazid son y1llar verem hastalığının tedavisinde kullanılan beş ilaçtan biridir (Tajudeen et al., 2013).

Etki mekanizması hücre (Shoeb et al., 1985; Zhang et al., 1992; Johnson et al., 1994) ve moleküler (Wahab et al., 2009) düzeyde incelenmiş izoniazid son yıllarda araştırmacıların dikkatini çekmektedir. İzoniazid türevlerinin antimikrobiyal etkinliğe sahip olduğu bilindikten (Bayrak et al., 2009) sonra çok sayıda izoniazid türevi sentezlenerek antimikobakteriyal, antiviral, antimikrobiyal aktiviteleri araştırılmaya başlandı (Slayden et al., 2000; Bottari et al., 2001; Maccari et al, 2002). Sentezlenen bazı isoniazid türevlerinin metal komplekslerinin de antimikrobiyal, antibakteriyal aktiviteleri test edildi (Prasad et al., 2009; Qurban, 2011; Kelode, 2013). Şu ana kadar bilinen izonikotinohidrazid türevi olan hidrazonların az bir kısmının kristal yapıları çözüldü (Yu et al., 2005; Naveenkumar et al., 2009; Bhat et al., 2012). Birçok metal iyonları ile kolayca dayanıklı kompleksler oluşturmalarından dolayı hidrazonlar anorganik kimyada önemli rol oynamaktadırlar. Biyoanorganik kimya alanındaki gelişmeler hidrazon komplekslerine ilgiyi artırd.

Sentezlenerek kristal yapısı çözülen ve $\mathrm{Cu}$, $\mathrm{Co}, \mathrm{Ni}$ ve $\mathrm{Zn}$ kompleksleri elde edilerek özellikleri incelenen hidrazonlardan biri de (1-naftilmetiliden) izonikotinohidrazid, $\mathrm{C}_{17} \mathrm{H}_{13} \mathrm{~N}_{3} \mathrm{O}(\mathrm{I})$, molekülüdür (Kriza et al, 2010). Ligandların koordinasyon bileşiklerinin yapısını ve özelliklerini büyük ölçüde belirlemesinden dolayı ligand olarak kullanılan moleküllerin moleküler düzeyde yapısal parametreleri, elektronik ve diğer fiziksel özelliklerinin araştııılması önem taşımaktadır. $\mathrm{Bu}$ çalışmada, değişik metallerle kompleksleri elde edilen ve moleküler düzeyde yanlızca kristal yapısı çözülen I molekülünün yapısal parametreleri, $\mu, \alpha, \beta$, $\mathrm{E}_{\text {номо }}, \mathrm{E}_{\text {LUмо }}$ ve $\Delta \mathrm{E}_{\mathrm{g}}=\mathrm{E}_{\text {LUмо }}-\mathrm{E}_{\text {номо }}$ ve değerleri DFT/ B3LYP ve HF metodlar1 ve $6-311++\mathrm{G}(\mathrm{d}, \mathrm{p})$ taban seti kullanılarak hesaplanmıştır. Çalışma sonucunda I molekülünün moleküler özellikleri belirlendi. Belirlenen bu önemli moleküler özellikler I ile yapılacak yeni çalışmalara yardımcı olunacaktır. Bu çalışmada incelenen I molekülünün kimyasal yapısı Şekil 1'de verilmiştir.

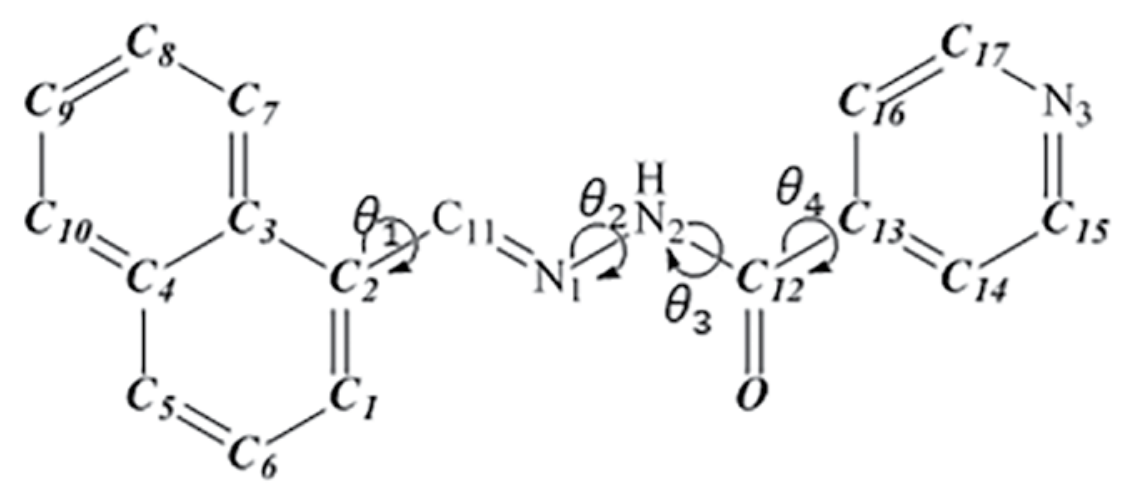

Şekil 1. (1-naftilmetiliden)izonikotinohidrazid moleklünün (I) kimyasal yapısı

\section{MATERYAL VE YÖNTEM}

\section{Hesaplama Yöntemleri}

Şekil 1'de kimyasal yapısı gösterilen I molekülünün üç boyuttaki (3D) yaklaşık geometrisi GaussView5.0 (Dennington et al., 2009) görüntüleme paket programında çizilerek Gaussian09 Rev B.01 paket programına (Frisch et al., 2010) giriş verisi olarak kullanıldı. I molekülünün konformasyon analizi B3LYP/6-311++G (d, p) yöntemi ile yapılarak, molekülün iki kararlı durum konformerleri (C-I ve $\mathrm{C}$-II) belirledi. C-I ve C-II konformerlerinin geometrik 
optimizasyonları ab initio metodu HF (Moller et al., 1934) ve DFT/Becke'nin 3 parametreli hibrit değiştokuş fonksiyoneli (B3) (Becket et al., 1988) ile LeeYang ve Parr'in korelasyon fonksiyonelinden (Lee et al., 1988; Beckel, 1993) oluşan B3LYP teori seviyesinde olmak üzere difüze ve polarize fonksiyonları içeren 6-311++G(d,p) temel seti (Franck et al., 1982; Rissoles et al., 2001) ile gaz fazında hesaplandı. C-I ve C-II konformerler için her iki modelde $\mu, \alpha, \beta, \mathrm{E}_{\text {номо }}$, $\mathrm{E}_{\text {LUMO }}$ ve $\Delta \mathrm{E}_{\mathrm{g}} 6-311++\mathrm{G}(\mathrm{d}, \mathrm{p})$ taban seti kullanılarak hesaplandi. Ayrıca, elde edilen optimize yapı ile GIAO yöntemine göre ${ }^{1} \mathrm{H}-\mathrm{NMR}$ ve ${ }^{13} \mathrm{C}-\mathrm{NMR}$ kimyasal kayma değerleri B3LYP/311++G(2d,p) ve HF/6-31G teori düzeylerinde (McLean et al., 1980; Krishnan et al., 1980) hesapland. Molekülün $\mathrm{E}_{\text {номо }}$ ve $\mathrm{E}_{\text {LUмо }}$ enerji değerlerini kullanarak aşağıdaki parametreler; iyonlaşma ptansiyel (IP $\approx-\mathrm{E}_{\text {номо }}$ ) lektron ilgisi $\left(\mathrm{EA} \approx-\mathrm{E}_{\mathrm{LUMO}}\right)$, elektronegatiflik $(\mathrm{\chi}=(\mathrm{IP}+\mathrm{EA}) / 2=$ $\left.-\left(\mathrm{E}_{\text {номо }}+\mathrm{E}_{\text {LUмо }}\right) / 2\right)$, moleküler sertlik $(\eta=(\mathrm{IP}-\mathrm{EA}) / 2$ $\left.\approx\left(\mathrm{E}_{\text {LUMO }}-\mathrm{E}_{\text {HOMO }}\right) / 2\right)$, kimyasal potansiyel $(\mu=-\chi), \mu=$ $($ EA- IP $\left.) / 2 \approx\left(\mathrm{E}_{\text {номо }}+\mathrm{E}_{\text {LUмо }}\right) / 2\right)$, elekrofilik indeksi( $\left.\omega=\mu^{2 / 2} \eta\right)$ ve kimyasal yumuşaklık $(S=1 / 2 \eta)$ ) ifadeleri ile hesaplanmıştır. Hesaplamalar sonucunda; polarizebilite ve hiperpolarizebilite değerlerinin kartezyen bileşenleri (a.u) elde edildi. Bu kartezyen bileşen değerlerinden polarizebilite;

$$
\alpha=\left(\frac{1}{3}\right)\left(\alpha_{x x}+\alpha_{y y}+\alpha_{z z}\right)
$$

Hiperpolarizebilite; $\beta$

$$
\beta=\left[\left(\beta_{x x x}+\beta_{x y y}+\beta_{x z z}\right)^{2}+\left(\beta_{y y y}+\beta_{y z z}+\beta_{y x x}\right)^{2}+\left(\beta_{z z z}+\beta_{z x x}+\beta_{z y y}\right)^{2}\right]^{1 / 2}
$$

eşitlikleri ile hesaplandı

\section{BULGULAR VE TARTIŞMA}

\section{Konformasyonel analiz}

Molekülün birden çok tek bağ merkezli dihedral açıya sahip olmasından dolayı bu bağlar etrafında serbest dönmeler olabileceğinden, bu tür esnek moleküllerin en kararlı durumunu bulmak için konformasyon analizi yapılarak oluşan konformasyonların enerji

$$
\mathrm{E}=\mathrm{E}_{\mathrm{PEY}}\left(\mathrm{R}_{\mathrm{i}}, \ldots \ldots \ldots \ldots \ldots \mathrm{R}_{\mathrm{n}}\right)
$$

Burada $\mathrm{R}_{\mathrm{i}}, \mathrm{N}$ atomlu doğrusal olmayan bir molekül sistemin potansiyel enerji yüzeyi (PEY) $3 \mathrm{~N}-6$, doğrusal molekül için $3 \mathrm{~N}-5$ tane koordinat boyutuna sahip olacaktır. Bu çalışmada I molekülünün konformasyon analizi, C3-C2-C1-N1, C11-N1$\mathrm{N} 2-\mathrm{C} 12, \quad \mathrm{~N} 1-\mathrm{N} 2-\mathrm{C} 12-\mathrm{C} 13$ ve N2-C12-C13-C14 dihedral açıları $10^{\circ}$ aralıklarla 36 adım değiştirilerek potansiyel enerji eğrileri, C3-C2-C11-N1 dihedral açısındaki her $10^{\circ}$ 'lik artış için C11- N1- N2- C12 dihedral açı $10^{\circ}$ aralıklarla 19 adım değiştirilerek PEY hesaplatıldı. Diğer dihedral açılar içinde (C11- ve geometrileri tespit edilir. Konformasyon analizi yapmanın amacı, molekülün potansiyel enerji yüzeyi (3D) ya da potansiyel enerji eğrisini (2D) belirleyerek, molekülün minimum enerjiye sahip olduğu kararlı denge (taban) durumunu belirlemektir. Bir potansiyel enerji yüzeyi (PEY), molekülün geometrisinin bir fonksiyonu olarak molekülün enerjisini veren matematiksel bir fonksiyondur: 


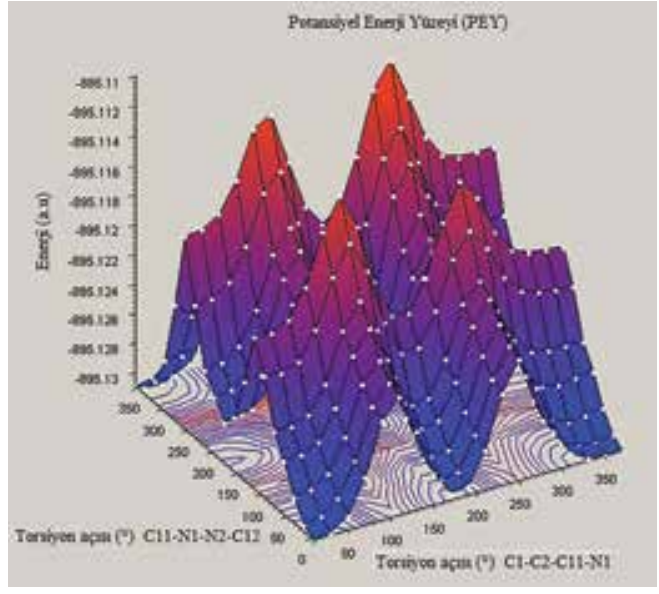

(a)

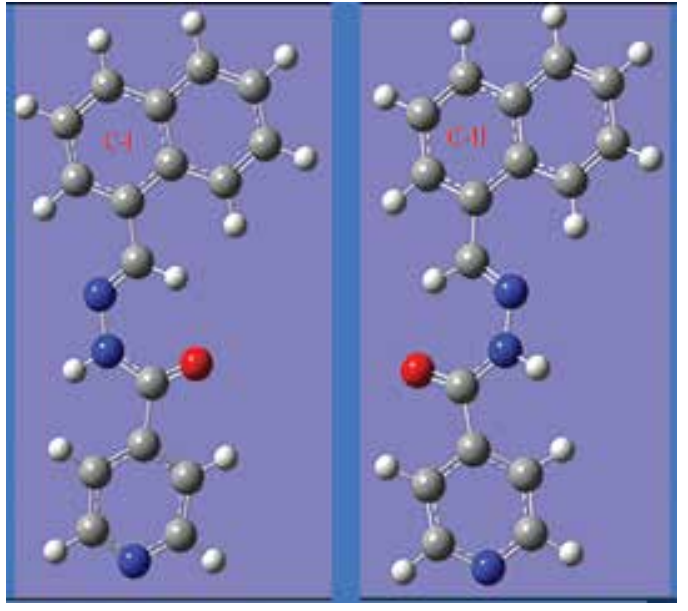

(b)

Şekil 2. I molekülünün (a) PEY, (b) optimize durumları

Ayrıca, molekülün konformasyon analizinde, hesaplandı. Hesaplama sonucu elde edilen PEY Şekil 3' C1-C2-C11-N1 torsiyon açıs1 C2-C11sigma $(\sigma)$ de verilmiştir. Şekil 3'den görüldüğü gibi, potansiyel bağ 1 etrafında $0^{\circ}$ den $360^{\circ}$ ye kadar $10^{\circ}$ lik artışlarla enerji eğrisinin $0^{\circ}, 180^{\circ}$ ve $360^{\circ}$ de minimum $90^{\circ}$ ve değiştirilerek molekülün potansiyel enerji yüzeyi (PEY) $\quad 270^{\circ}$ de ise maksimum enerjiye sahiptir.

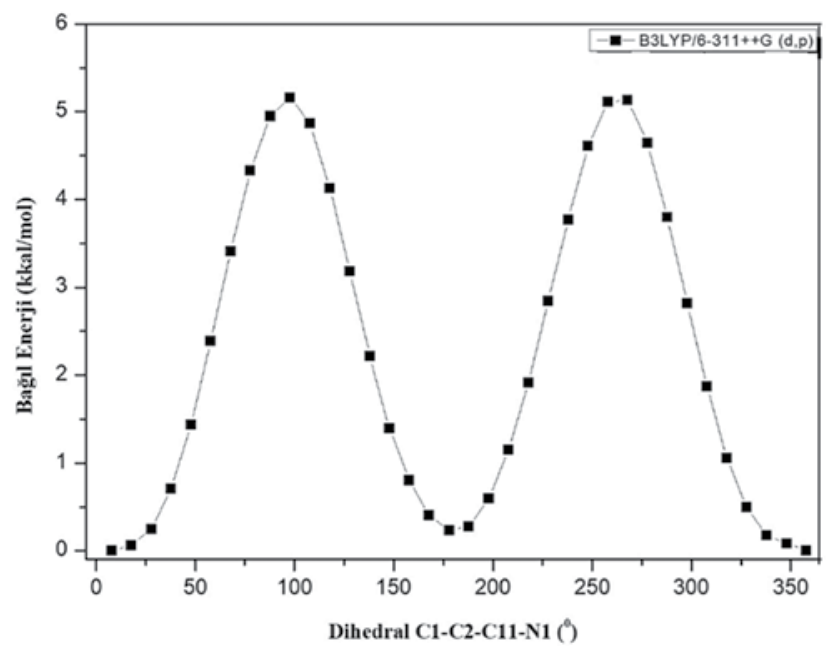

Şekil 3. I molekülünün potansiyel eğrisi

\section{Geometrik Optimizasyon}

I molekülün, $\mathrm{C}_{17} \mathrm{H}_{13} \mathrm{~N}_{3} \mathrm{O}$, yapılan konformasyon analizi sonucu elde edilen genel ve yerel minimum yapıları HF ve DFT/B3LYP metodu ve polarize fonksiyonları içeren $6-311 \mathrm{G}++(\mathrm{d}, \mathrm{p})$ temel seti ile optimize edildi. Yapılan optimizasyonlar sonucunda genel ve yerel minimum yapıların C-I ve C-II konformerlerine dönüştüğü, C-I konformerinin C-II konformerinden daha kararlı olduğu görüldü. $\mathrm{Bu}$ konformerlerin optimize edilmiş yapıları Şekil 2(b)'de verildi. C-I ve C-II konformerleri arasındaki enerji fark1 DFT: $0.25368 \mathrm{kkal} / \mathrm{mol}$, HF:0.53313 kkal $/ \mathrm{mol}$ olarak hesaplandı. Her iki konformer için hesaplanan geometrik parametreler, molekülün deneysel sonuçları (Kriza et al, 2010) ile karşılaştırmalı olarak bağ uzunlukları Çizelge 1, bağ ve dihedral açıları Çizelge 2'de verilmiştir. Teorik ve deneysel geometrik parametreleri sağlıklı bir şekilde karşılaştırmak için, bağ uzunluklarına ait korelasyon grafikleri Şekil 4 (a), (b)‘de verilmiştir. Bu grafiklerden de görüldüğü gibi molekülün deneysel bağ uzunlukları teorik yöntemle hesaplanan bağ uzunlukları ile uyum içindedir. 
Çizelge 1. I molekülün B3LYP ve HF yöntemlerine ile elde edilen bağ uzunlukları ( $\AA$ )

\begin{tabular}{|c|c|c|c|c|c|}
\hline \multirow{2}{*}{$\begin{array}{l}\text { Parametreler } \\
\text { Bağlar }\end{array}$} & \multicolumn{2}{|c|}{ C-I konformer } & \multicolumn{3}{|c|}{ C-II konformer } \\
\hline & HF & B3LYP & Deney $^{\mathbf{a}}$ & HF & B3LYP \\
\hline $\mathrm{C} 1-\mathrm{C} 2$ & 1.3642 & 1.3867 & $1.3672(2)$ & 1.3672 & 1.3898 \\
\hline C1-C6 & 1.4108 & 1.4048 & $1.402(2)$ & 1.4116 & 1.4068 \\
\hline $\mathrm{C} 2-\mathrm{C} 3$ & 1.4319 & 1.4384 & $1.434(2)$ & 1.4403 & 1.4426 \\
\hline C2-C11 & 1.4825 & 1.4691 & $1.4693(2)$ & 1.4791 & 1.4672 \\
\hline $\mathrm{C} 3-\mathrm{C} 4$ & 1.4108 & 1.4351 & $1.429(2)$ & 1.4124 & 1.4353 \\
\hline $\mathrm{C} 3-\mathrm{C} 7$ & 1.424 & 1.4227 & $1.418(2)$ & 1.4214 & 1.4202 \\
\hline $\mathrm{C} 4-\mathrm{C} 5$ & 1.418 & 1.4167 & $1.408(3)$ & 1.4211 & 1.4194 \\
\hline $\mathrm{C} 4-\mathrm{C} 10$ & 1.4204 & 1.4206 & $1.422(2)$ & 1.4182 & 1.4191 \\
\hline $\mathrm{C} 5-\mathrm{C} 6$ & 1.3576 & 1.3756 & $1.354(3)$ & 1.3537 & 1.3720 \\
\hline $\mathrm{C} 7-\mathrm{C} 8$ & 1.3587 & 1.3750 & $1.368(3)$ & 1.3615 & 1.3775 \\
\hline C9-C10 & 1.3568 & 1.3722 & $1.352(3)$ & 1.3577 & 1.3734 \\
\hline C8-C9 & 1.4145 & 1.4128 & $1.400(3)$ & 1.4109 & 1.4104 \\
\hline C11-N1 & 1.2571 & 1.2877 & $1.2680(2)$ & 1.2570 & 1.2883 \\
\hline N1-N2 & 1.3721 & 1.3722 & $1.3781(6)$ & 1.3794 & 1.3752 \\
\hline $\mathrm{N} 2-\mathrm{C} 12$ & 1.3596 & 1.3749 & $1.3567(2)$ & 1.3638 & 1.3746 \\
\hline C12-C13 & 1.5077 & 1.5074 & $1.5071(2)$ & 1.5077 & 1.5085 \\
\hline $\mathrm{C} 12-\mathrm{O}$ & 1.1943 & 1.2219 & $1.2158(2)$ & 1.1924 & 1.2215 \\
\hline C14-C15 & 1.3836 & 1.3914 & $1.384(2)$ & 1.3837 & 1.3915 \\
\hline C15-N3 & 1.3205 & 1.3377 & $1.333(2)$ & 1.3205 & 1.3377 \\
\hline C13-C14 & 1.3849 & 1.3958 & $1.386(2)$ & 1.3849 & 1.3957 \\
\hline $\mathrm{C} 13-\mathrm{C} 16$ & 1.3858 & 1.3973 & $1.383(2)$ & 1.3859 & 1.3973 \\
\hline $\mathrm{C} 16-\mathrm{C} 17$ & 1.3862 & 1.3940 & $1.388(2)$ & 1.3861 & 1.3940 \\
\hline C17-N3 & 1.3174 & 1.3348 & $1.338(2)$ & 1.3175 & 1.3348 \\
\hline RMS & 0.009971 & 0.011126 & & 0.009792 & 0.011371 \\
\hline
\end{tabular}

a: Kriza et al., 2010

Ayrıca elde edilen bağ uzunluklarına ait değerlerin RMS (Karelerin ortalamasının karekökü) değerleri hesaplanarak Çizelge 1 'in alt satırında verilmiştir. RMS değerleri aşağıdaki eşitliğe göre hesaplanmıştır. Burada bağ uzunluğu olarak alındı.

$$
R M S=\sqrt{\frac{\sum\left(X_{d e n}-X_{t e o}\right)^{2}}{N}}
$$

Çizelge 1'den görüldüğü gibi I molekülünün her iki konformeri için $\mathrm{HF} / 6-311++\mathrm{G}(\mathrm{d}, \mathrm{p})$ metodunda hesaplanan RMS değerler B3LYP/6-311++G(d,p) metodunda hesaplanan değerlerden daha küçüktür. $\mathrm{Bu}$ da $\mathrm{HF} / 6-311++\mathrm{G}(\mathrm{d}, \mathrm{p})$ metodunda hesaplanan değerlerin deneysel değerlerle daha uyumlu olduğu anlamina gelmektedir. 
Çizelge 2. I molekülünün B3LYP ve HF yöntemleri ile elde edilen bağ açıları ( ${ }^{\circ}$ )

\begin{tabular}{lcllll}
\hline Parametreler & \multicolumn{2}{c}{ C-I konformer } & & \multicolumn{2}{l}{ C-II konformer } \\
\hline Bağ açıları ( ${ }^{\circ}$ ) & HF & B3LYP & Deney $^{\mathbf{a}}$ & HF & B3LYP \\
\hline C1-C2-C11 & 120.1 & 120.2 & 119.9 & 126.6 & 127 \\
C2-C11-N1 & 119.9 & 119.9 & 121.1 & 124.9 & 123.9 \\
C11-N1-N2 & 120.4 & 120.7 & 118.5 & 118.3 & 120.5 \\
N2-C12-C13 & 114.2 & 114.2 & 115.2 & 114.2 & 114.1 \\
N2-C12-O & 124.7 & 124.3 & 124.0 & 124.6 & 124.4 \\
C12-C13-C16 & 118.2 & 118.0 & 118.4 & 118.2 & 117.9 \\
C12-C13-C14 & 123.4 & 124.1 & 123.2 & 123.4 & 124.2 \\
Dihedral açlarl ( $\left.{ }^{\circ}\right)$ & & & & & \\
\hline C1-C2-C11-N1 & 30.5 & 7.8 & - & 178.7 & -178.1 \\
C11-N1-N2-C12 & -29.9 & -4.4 & - & -44.9 & -2.9 \\
N1-N2-C12-C13 & -171.5 & 179.2 & - & -168 & 178.7 \\
N2-C12-C13-C16 & 149.2 & 152.1 & - & 150.4 & 152.4 \\
N2-C12-C13-C14 & -32.3 & -29.9 & - & -31 & -29.6 \\
\hline
\end{tabular}

a: Kriza et al., 2010

Bağ açıları için hesaplanan değerlerle deneysel değerler karşılaştırıldığında, C-I konformerine ait

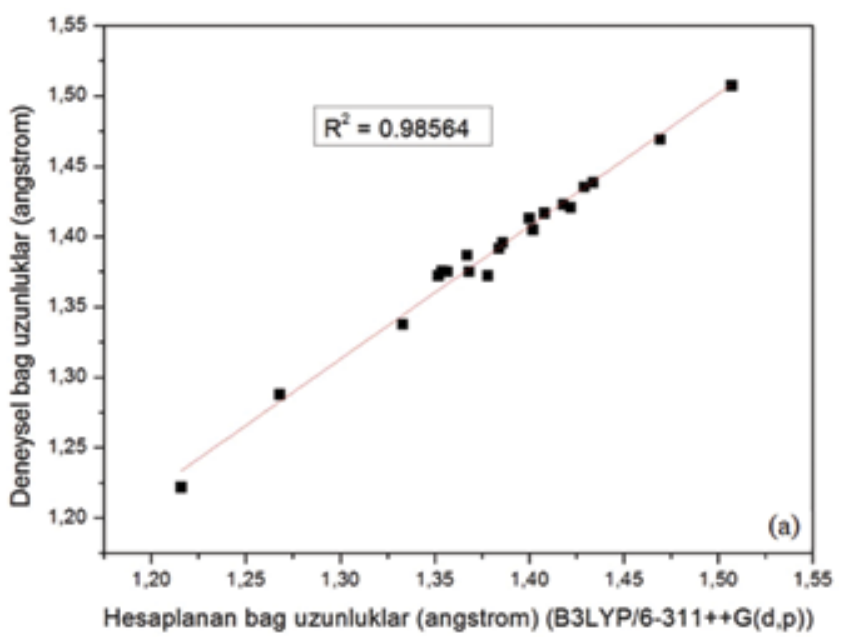

olan değerlerin daha uyumlu olduğu Çizelge 2'den de görülmektedir.

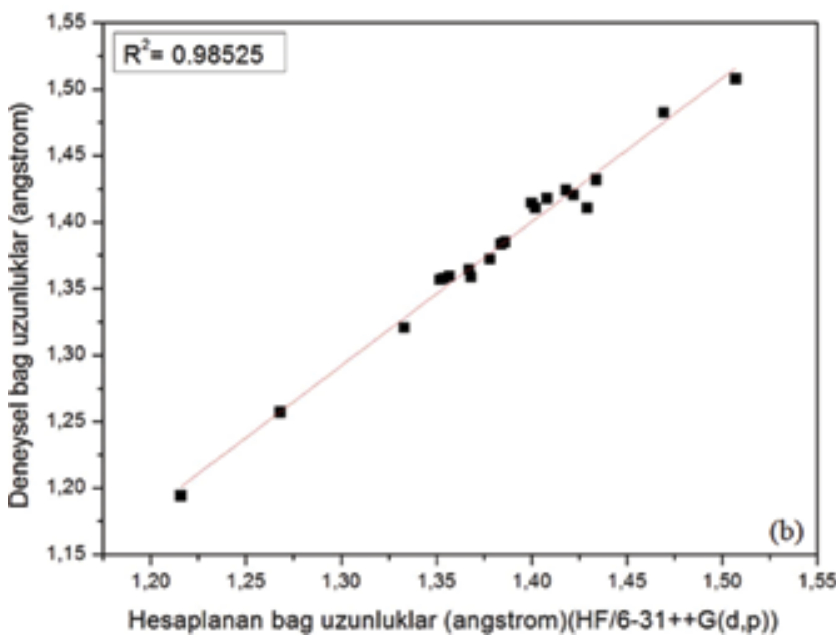

Şekil 4. I molekülün deneysel ve teorik bağ uzunluklarının korelasyonu

HOMO-LUMO Enerjileri ve Elektronik hesaplandi. Hesaplamalar sonucundan elde edilen Özelliklerinin İncelenmesi

I molekülünün $\mathrm{C}$-I ve $\mathrm{C}$-II konformerlerinin elektronik enerji, $\mu, \alpha, \beta, \mathrm{E}_{\text {номо }}$ ve $\mathrm{E}_{\text {LUмо }}$ her iki model için de $6-311++\mathrm{G}(\mathrm{d}, \mathrm{p})$ taban seti kullanılarak elektronik enerji, $\mu, \alpha, \beta, \mathrm{E}_{\text {LUмо }}-\mathrm{E}_{\text {номо }}$ değerleri Çizelge 3'de verildi. Ayrıca ve en yüksek dolu orbitaller (HOMO) ve en düşük boş orbitaler (LUMO)'nun 3D çizimi Şekil 5'de verildi. 
Çizelge 3. I molekülün elektronik enerji, $\mu, \alpha, \beta, \mathrm{E}_{\text {LUмо }}-\mathrm{E}_{\text {номо }}$ değerleri

\begin{tabular}{lllll}
\hline & C-I konformer & \multicolumn{3}{l}{ C-II konformer } \\
\hline Parametreler & HF & DFT & HF & DFT \\
\hline$\mu(\mathrm{D})$ & 1.94 & 2.05 & 2.32 & 2.32 \\
$\alpha($ a.u $)$ & 173.01 & 251.98 & 217.45 & 252.06 \\
$\beta($ a.u. $)$ & 114.30 & 2634.83 & 373.43 & 2675.28 \\
$\beta\left(10^{-30}\right.$ esu) & - & 22.76 & - & 23.11 \\
$\Delta$ E(HOMO-LUMO) (eV) & 8.90 & 3.67 & 8.97 & 3.76 \\
Elektronik enerji (a.u) & -889.72767029 & -895.32980707 & -889.72682070 & -895.32940280 \\
\hline
\end{tabular}

Moleküler sistemlerin NLO özelliklerinin belirlenmesinde referans olarak, genellikle üre molekülünün NLO değeri kullanılır (Sangeetha et al., 2014).
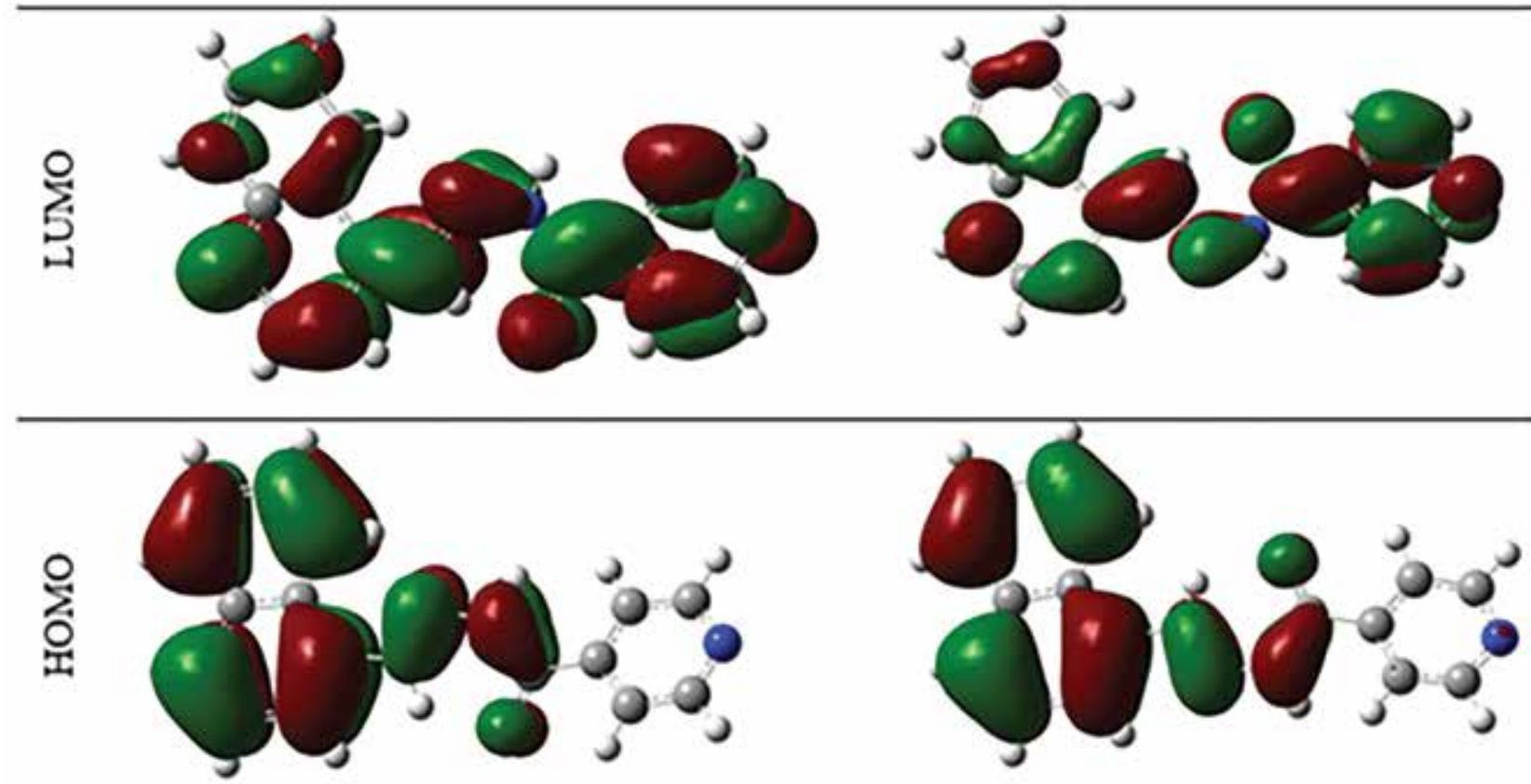

\section{C-I konformer}

\section{C-II konformer}

3

Şekil 5. Molekülün en yüksek dolu orbitalleri ve en düsük boş orbitallerinin 3D çizimi

$\mathrm{E}_{\text {номо }}$ ve $\mathrm{E}_{\text {LUмо }}$ enerjilerinden molekülün sınır moleküler enerji aralığı, Kimyasal potansiyel $\mu$, Elekrofilik indeksi $\omega$, İyonlaşma Potansiyeli IP, Elektron İlgisi EA, Elektronegatiflik $\chi$, Moleküler Yumuşaklık S, Moleküler Sertlik $\eta$ gibi elektronik özellikleri hesaplanarak, Çizelge 4'de verildi. Hesaplama sonuçlarına göre C-I ve C-II konformerlerinin birinci-derece hiperpolarizebilite değerleri üre molekülünkinden yaklaşık olarak, sırasıyla, 29 ve 30 kez daha büyüktür (B3LYP / 6-311 ++ G (d, p) için; üre $\beta=0.7803 \times 10^{-30} \mathrm{esu}, 1$ a.u. $=8,6393 \times 10^{-33} \mathrm{esu}$ ). 
Çizelge 4. I molekülün elektronik özellikleri

\begin{tabular}{lllll}
\hline & \multicolumn{2}{c}{ C-I konformer } & \multicolumn{2}{c}{ C-II konformer } \\
\hline Parametreler & HF & DFT & HF & DFT \\
\hline $\mathrm{E}_{\text {Hомо (a.u) }}$ & -0.2932 & -0.2221 & -0.2954 & -0.2249 \\
$\mathrm{E}_{\text {LUMO (a.u) }}$ & 0.0340 & -0.0873 & 0.0342 & -0.0869 \\
$\mathrm{E}_{\text {Hомо }}(\mathrm{eV})$ & -7.9789 & -6.0440 & 8.0390 & -6.1196 \\
$\mathrm{E}_{\text {Luмо }}(\mathrm{eV})$ & 0.9242 & -2.3758 & -0.9300 & -2.3640 \\
$\mathrm{E}_{\text {Lumo }} \mathrm{E}_{\text {HOмо }}(\mathrm{eV})$ & 8.9031 & 3.6682 & 8.9689 & 3.7556 \\
İyonlaşma Potansiyeli IP(eV) & 7.9789 & 6.0440 & 8.0390 & 6.1196 \\
Elektron İlgisi EA $(\mathrm{eV})$ & -0.9242 & 2.3758 & -0.9300 & 2.3640 \\
Elektronegatiflik $\chi(\mathrm{eV})$ & 3.5274 & 4.2099 & 3.5545 & 4.2418 \\
Moleküler Sertlik $\eta(\mathrm{eV})$ & 4.4515 & 1.8341 & 4.4845 & 1.8778 \\
Moleküler Yumuşaklık S(eV) & 0.1123 & 0.2726 & 0.1115 & 0.2663 \\
Kimyasal potansiyel $\mu(\mathrm{eV})$ & -3.5274 & -4.2099 & 3.5545 & -4.2418 \\
Elekrofilik indeksi $\omega(\mathrm{eV})$ & 1.3975 & 4.8317 & 1.4087 & 4.7910 \\
\hline
\end{tabular}

\section{NMR ve IR Spektral Analiz}

I molekülünün C-I konformer yapısının ${ }^{13} \mathrm{C}$ ve ${ }^{1} \mathrm{H} \quad$ ve $\mathrm{HF} / 6-31 \mathrm{G}(\mathrm{d})$ yöntemleri ile hesaplandi ve sonuçlar NMR kimyasal kayma değerleri B3LYP/6-311+G(2d,p) Çizelge 5'de verildi.

Çizelge 5. I molekülün ${ }^{13} \mathrm{C}$ ve ${ }^{1} \mathrm{H}$ NMR kimyasal kayma değerleri

\begin{tabular}{|c|c|c|c|c|c|c|c|c|c|c|c|}
\hline \multicolumn{6}{|c|}{ B3LYP/6311+G (2d,p) } & \multicolumn{6}{|c|}{ HF/6-31G(d) } \\
\hline Atom & $(\mathbf{p p m})$ & Atom & $(\mathbf{p p m})$ & Atom & ppm) & Atom & (ppm) & Atom & (ppm) & Atom & ppm) \\
\hline $\mathrm{C} 12$ & 187.62 & $\mathrm{C} 7$ & 144.85 & $\mathrm{C} 11-\mathrm{H}$ & 11.86 & $\mathrm{C} 12$ & 158.85 & $\mathrm{C} 7$ & 120.14 & $\mathrm{C} 11-\mathrm{H}$ & 9.99 \\
\hline $\mathrm{C} 17$ & 175.79 & $\mathrm{C} 1$ & 144.84 & $\mathrm{C} 17-\mathrm{H}$ & 9.22 & $\mathrm{C} 17$ & 147.82 & $\mathrm{C} 1$ & 123.30 & $\mathrm{C} 17-\mathrm{H}$ & 8.92 \\
\hline $\mathrm{C} 15$ & 173.96 & $\mathrm{C} 16$ & 144.73 & $\mathrm{C} 15-\mathrm{H}$ & 9.10 & $\mathrm{C} 15$ & 146.49 & $\mathrm{C} 16$ & 118.65 & $\mathrm{C} 15-\mathrm{H}$ & 8.82 \\
\hline $\mathrm{C} 11$ & 171.33 & $\mathrm{C} 14$ & 138.41 & $\mathrm{C} 1-\mathrm{H}$ & 8.82 & $\mathrm{C} 11$ & 149.12 & $\mathrm{C} 14$ & 113.80 & $\mathrm{C} 7-\mathrm{H}$ & 8.29 \\
\hline $\mathrm{C} 13$ & 165.14 & & & $\mathrm{~N}-\mathrm{H}$ & 8.79 & $\mathrm{C} 13$ & 141.15 & & & $\mathrm{C} 1-\mathrm{H}$ & 8.13 \\
\hline $\mathrm{C} 4$ & 157.37 & & & $\mathrm{C} 9-\mathrm{H}$ & 8.72 & $\mathrm{C} 4$ & 129.18 & & & $\mathrm{C} 5-\mathrm{H}$ & 7.87 \\
\hline $\mathrm{C} 3$ & 156.04 & & & $\mathrm{C} 5-\mathrm{H}$ & 8.22 & $\mathrm{C} 3$ & 129.44 & & & $\mathrm{C} 10-\mathrm{H}$ & 7.81 \\
\hline $\mathrm{C} 2$ & 154.28 & & & C16-H & 8.18 & $\mathrm{C} 2$ & 126.68 & & & C16-H & 7.78 \\
\hline $\mathrm{C} 5$ & 153.77 & & & C10-H & 8.17 & $\mathrm{C} 5$ & 127.61 & & & $\mathrm{C} 8-\mathrm{H}$ & 7.59 \\
\hline $\mathrm{C} 10$ & 151.31 & & & C8-H & 7.92 & $\mathrm{C} 10$ & 125.18 & & & C9-H & 7.45 \\
\hline $\mathrm{C} 8$ & 148.64 & & & C6-H & 7.77 & C8 & 123.55 & & & C6-H & 7.42 \\
\hline C9 & 147.55 & & & C9-H & 7.75 & C9 & 121.86 & & & C14-H & 7.17 \\
\hline C6 & 146.85 & & & C14-H & 7.44 & C6 & 120.93 & & & $\mathrm{~N}-\mathrm{H}$ & 6.82 \\
\hline
\end{tabular}


Her iki hesaplama yönteminde de molekülün en yüksek kimyasal kayma değerine karbonil grubunun karbon atomu C12'nin sahip olduğu, sirasiyla bu değerlerin $187.62 \mathrm{ppm}$ ve $158.85 \mathrm{ppm}$ olduğu gözlenmişdir. C12 karbon atomunun kimyasal kayma değerinin büyük çıkması karbonil grubunun (daha elektronegatif ) oksijen atomuna bağlı olması ile izah edilebilinir. Piridin halkasındaki azot atomuna bağlı olan C17 ve C15 karbon atomlarının molekülde bulunan diğer karbon atomlarının kimyasal kayma değerinden daha büyük değere sahip oldukları görüldü. En küçük kimyasal kayma değerine ise piridin halkasındakidaki $\mathrm{C} 16$ ve $\mathrm{C} 14$ karbon atomlarının sahip olduğu gözlenmişdir. $\mathrm{Bu}$ değerler sirasiyla $144.73 / 138.41 \mathrm{ppm}$ ve
118.65/113.80 ppm'dir. Çizelge 5'den görüldüğü gibi piridin ve naftalin halklarına bağlı olmayan C11-H) protonunun en yüksek değere (sırasıyla 11.86 ppm ve 9.99 ppm) sahip olduğu gözlendi. I molekülünün 34 atoma sahip olmasindan dolayı düzlem içi ve dışı olmak üzere 96 titreşim frekansına sahiptir. $\mathrm{Bu}$ çalışmada I molekülünün titreşim frekanslar1 B3LYP/6-311++G(d,p) teori düzeyinde hesaplatıldı ve sonuçlar seçilmiş yöntem için 0.963 skala faktörü ile çarpılarak uyumlu hale getirilerek, bazı gerilme titreşimleri Çizelge 6'da verildi. Genelde C-H gerilme titreşimi 3000-3100 $\mathrm{cm}^{-1}$ aralığında gözlenir, hesaplama sonuçlarında bu frekans aralığı yaklaşı $3036-3098 \mathrm{~cm}^{-1}$ olarak görüldü.

Çizelge 6. I molekülünün seçilmiş titreşim değerleri

\begin{tabular}{|c|c|c|c|}
\hline \multirow[t]{2}{*}{ Titreşim molar } & \multicolumn{2}{|c|}{ Teorik/B3LYP/6-311++G***(cm- $\left.{ }^{-1}\right)$} & \multirow{2}{*}{$\frac{\text { Deneysel }\left(\mathrm{cm}^{-1}\right)}{\text { Deney }^{\mathrm{a}}}$} \\
\hline & Skala edilmemiş & Skala edilmiş & \\
\hline$v \mathrm{NH}(100)$ & 3639,473 & 3504.813 & 3175 \\
\hline$v \mathrm{CH}(24)$ & 3217,181 & 3098.145 & - \\
\hline$v \mathrm{CH}(34)$ & 3205,202 & 3086.609 & - \\
\hline$v \mathrm{CH}(49)$ & 3199,679 & 3081.291 & - \\
\hline$v \mathrm{CH}(27)$ & 3197,406 & 3079.102 & - \\
\hline$v \mathrm{CH}(66)$ & 3185,995 & 3068.113 & - \\
\hline$v \mathrm{CH}(41)$ & 3180,67 & 3062.985 & - \\
\hline vCH (64) & 3179,992 & 3062.333 & - \\
\hline$v \mathrm{CH}(65)$ & 3171,436 & 3054.093 & - \\
\hline$v \mathrm{CH}(43)$ & 3162,564 & 3045.549 & - \\
\hline$v \mathrm{CH}(42)$ & 3159,186 & 3042.297 & - \\
\hline$v \mathrm{CH}(54)$ & 3158,078 & 3041.229 & - \\
\hline$v \mathrm{CH}(56)$ & 3152,946 & 3036.287 & - \\
\hline$v \mathrm{CO}(44)$ & 1723,642 & 1659.867 & 1675 \\
\hline$v \mathrm{CN}(30)$ & 1629,9127 & 1569,606 & 1552 \\
\hline
\end{tabular}

Aynı şekilde N-H gerilme hareketi $3175 \mathrm{~cm}^{-1}, \mathrm{C}=\mathrm{O}$ $1675 \mathrm{~cm}^{-1}$ aralığında gözlenir iken (Kriza et al., 2010), hesaplama sonuçlarında bu gerilme frekansları 3504 $\mathrm{cm}^{-1}$ ve $1659 \mathrm{~cm}^{-1}$ olarak elde edildi. 


\section{SONUÇLAR}

(1-naftilmetiliden)izonikotinohidrazid molekülünün konformasyon analizi yapılarak, molekülün iki kararlı durum konformerleri (C-I ve C-II) belirledi. C-I ve $\mathrm{C}$-II konformerlerinin geometrik optimizasyonları $a b$ initio metodu Hartree Fock metodu ve yoğunluk fonksiyoneli teoris ile $6-311++\mathrm{G}(\mathrm{d}, \mathrm{p})$ temel seti kulanılarak hesaplandı. C-I konformerinin hesaplanan bağ uzunlukları ile deneysel değerler arasında regresyon analizi yapılarak, B3LYP metodundaki uyumluluk $\mathrm{R}^{2}=0.98564$, HF $\mathrm{R}^{2}=0.98525$ olarak bulundu. I molekülünün C-I ve C-II konformerlerinin dipol momenti, polarizebilite, birinci-derece

\section{KAYNAKLAR}

Bayrak H, Demirbaş A, Demirbaş N, Karaoğlu SA, 2009. Synthesis of some new 1, 2, 4-triazoles starting from isonicotinic acid hydrazide and evaluation of their antimicrobial activities. European Journal of Medicinal Chemistry, 44: 4362-4366.

Becke AD, 1988. Density-functional exchange-energy approximation with correct asymptotic behavior. Physical Review A, 38(6):3098-310

Becke AD, 1993 Density-functional thermochemistry 3. the role of exact exchange. The Journal of Chemical Physics, 98 (7): 5648-5652.

Bhat MA, Abdel-Aziz HA, Ghabbour HA, Hemamalini M, Fun HK, 2012. (E)-N'-(4-Isopropylbenzylidene)isonicotinohydrazide monohydrate. Acta Crystallographica, 68: 1002.

Bloom BR, Murray CJL, 1992. Tuberculosis: Commentary on a reemergent killer. Science, 257: 1055-1064.

Bottari B, Maccari R, Monforte F, Ottanà R, Vigorita MG, Bruno G, Nicolò F, Rotondo A, Rotondo E, 2001. Nickel (II) 2, 6-diacetylpyridine bis (isonicotinoylhydrazonate) and bis (benzoylhydrazonate) complexes: structure and antimycobacterial evaluation. Part XI. Bioorganic \& Medicinal Chemistry, 9: 2203-2211.

Dennington R, Keith T, Millam J, 2009. Semichem Inc., GaussView, Version 5, Shawnee Mission KS,

Francl MM, Pietro WJ, Hehre WJ, Binkley JS, Gordon, MS, DeFrees DJ, Pople, JA, 1982. Self-consistent molecular orbital methods. XXIII. A polarization-type basis set for second-row elements. Chemical Physics, 77: 3654-3665

Frisch MJ, Trucks GW, Schlegel HB, Scuseria GE, Robb MA, Cheeseman JR, Scalmani G, Barone V, Mennucci B, Petersson GA, Nakatsuji H, Caricato M, Li X, Hratchian HP, Izmaylov AF, Bloino J, Zheng G, Sonnenberg JL, Hada M, Ehara M, Toyota K, Fukuda R, Hasegawa J, Ishida M, Nakajima T, Honda Y, Kitao O, Nakai H, Vreven T, Montgomery JA, Vreven TJ., Peralta JE, Ogliaro F, Bearpark M, Heyd JJ, Brothers E, Kudin N, Staroverov VN, Kobayashi R, Normand J, Raghavachari K, Rendell A, Burant JC, Iyengar SS, Tomasi J, Cossi M, Rega N, Millam JM, Klene M, Knox JE, Cross JB, Bakken V, Adamo C, Jaramillo J, Gomperts R, Stratmann RE, Yazyev O, Austin AJ, Cammi R, Pomelli CJ, Ochterski hiperpolarizebilite $(\beta)$, en yüksek dolu molekül orbital, en düşük boş molekül orbital enerjileri her iki yöntem ile hesaplandi. Bunlara ek olarak, hesaplanmış $\mathrm{E}_{\text {номо }}$ ve $\mathrm{E}_{\text {LUMO }}$ enerjileri kullanılarak molekülün sınır moleküler enerji aralı̆ $\breve{1}$, Kimyasal potansiyel $\mu$, Elektrofili indeksi $\omega$, İyonlaşma Potansiyeli IP, Elektron İlgisi EA, Elektronegatiflik $\chi$, Moleküler Yumuşaklık S, Moleküler Sertlik $\eta$ gibi elektronik özellikleri elde edildi. Ayrıca teorik hesaplamalar sonucu elde edilen birinci-derece hiperpolarizebilite değerleri, üre molekülünkinden çok büyük olduğu belirlendi. Bu özellik molekülün potansiyel bir NLO materyal adayı olduğunu ve optik materyal olarak kullanılabileceğini göstermektedir.

W, Martin LR, Morokuma K, Zakrzewski VG, Voth GA, Salvador P, Dannenberg JJ, Dapprich S, Daniels AD, Farkas O, Foresman JB, Ortiz JV, Cioslowski J, Fox D J, 2010. Gaussian Inc., (Wallingford, CT).

Johnson K, Schultz PG, 1994. Mechanistic studies of the oxidation of isoniazid by the catalase peroxidase from Mycobacterium tuberculosis. Journal of the American Chemical Society, 116: $7425-7426$

Judge V, Narasimhan B, Ahuja M, Sriram D, Yogeeswari P, De Clercq E, Pannecouque C, Balzarni J, 2012. Isonicotinic acid hydrazide derivatives: synthesis, antimicrobial activity, and QSAR studies. Medicinal Chemistry Research, 21: 14511470.

Kelode SR, 2013. Thermal and antibacterial studies of cobalt(II), nickel(II), copper(II) and zinc(II) complexes of hydrazone Schiff base. Journal of Chemical and Pharmaceutical Research, 5(6): 60-63.

Krishnan R, Binkley JS, Seeger R, Pople JA, 1980. Self-consistent molecular-orbital methods. basis set for correlated wavefunctions, The Journal of Chemical Physics, 72: 650-654.

Kriza A, Ababei LV, Cioatera N, Rău I, Stănică N, 2010. Synthesis and structural studies of complexes of $\mathrm{Cu}, \mathrm{Co}, \mathrm{Ni}$ and $\mathrm{Zn}$ with isonicotinic acid hydrazide and isonicotinic acid (1-naphthylmethylene)hydrazide. Journal of the Serbian Chemical Society, 75(2): 229-242.

Lee CT, Yang WT, Parr RG, 1988. Development of the colle-salvetti correlation-energy formula into a functional of the electron density. Physical Review B, 37: 785-789.

Maccari R, Ottanà R, Monforte F, Vigorita MG, 2002. InVitro antimycobacterial activities of 2-monosubstituted isonicotinohydrazides and their cyanoborane adducts. Antimicrobial Agents and Chemotherapy, 46: 294-299.

McLean AD, Chandler GS, 1980. Contracted Gaussian basis sets for molecular calculations. I. Second row atoms, $Z=11-18$. The Journal of Chemical Physics, 72: 5639-5648.

Moller C, Plesset MS, 1934. Note on an approximation treatment for many- electron systems. Physical Review, 46: 618-622.

Naveenkumar HS, Sadikun A, Ibrahim P, Loh WS, Fun HK, 2009. (E)-N'-(2,4,5-Trimethoxybenzylidene)isonicotinohydrazide dihydrate. Acta Crystallographica, 65: 2540-2541. 
Prasad S, Agarwal RK, 2009. Synthesis, physico-chemical and biological Properties of complexes of cobalt(II) derived from hydrazones of isonicotinic acid hydrazide Journal of the Korean Chemical Society, 53: 17-26.

Qurban SW, 2011. Synthesis and characterization of some transition metal complexes of Schiff base derived from isonicotinic hydrazide and O-Vanillin. Diyala Journal for Pure Sciences, 7(2): 94-104.

Rassolov VA, Ratner MA, Pople JA, Redfern PC, Curtiss LA, 2001. 6-31G* basis set for third-row atoms. Journal of Computational Chemistry, 22: 976-984.

Sangeetha CC, Madivanane R, Pouchaname V, Vijaya Prasath R, 2014. Experimental (FT-IR \& FT - Raman) and theoretical investigation, electronic properties of quinoxaline. International Journal of ChemTech Research, 6 (5): 28542865.

Sbarbaro JA, 1997. Multidrug-resistant tuberculosis. It is time to focus on the private sector of medicine. Chest, 111: 11491151.

Slayden RA, Barry CE, 2000. The genetics and biochemistry of isoniazid resistance in mycobacterium tuberculosis. Microbes and Infection, 2: 659-669.
Shoeb HA, Bowman BU, Ottoleghi AC, Merola AJ, 1985. Peroxidase-mediated oxidation of isoniazıd. Antimicrobial Agents and Chemotherapy, 27: 399-403.

Sriram D, Yogeeswari P, Priya DY, 2009. Antimycobacterial activity of novel $\mathrm{N}$-(substituted)-2-isonicotinoylhydrazinocarbothioa mide endowed with high activity towards isoniazid resistant tuberculosis. Biomedicine \& Pharmacotherapy, 63: 36-39.

Tajudeen SS, Kannappan G, 2013. Synthetic, structural and pharmacological studies on some isonicotinohydrazide and benzohydrazide analogues. Journal of Pharmacy Research, 7: 534-539.

Yu M, Chen X, Jing ZL, 2005. Isonicotinic acid (2-hydroxy-3-methoxybenzylidene) hydrazide. Acta Crystallographica, 61: 13451346.

Wahab HA, Choong YS, Ibrahim P, Sadikun A, Scior T, 2009. Elucidating Isoniazid Resistance Using Molecular Modeling. Journal of Chemical Information and Modeling, 49: 97-107.

Zhang Y, Heym B, Allen B, Young D, Cole S, 1992. The catalaseperoxidase gene and isoniazid resistance of Mycobacterium tuberculosis. Nature, 358: 591-593. 